\title{
Experimental estimation of the dimension of classical and quantum systems
}

\author{
Martin Hendrych ${ }^{1}$, Rodrigo Gallego ${ }^{1}$, Michal Mičuda ${ }^{1,2}$, Nicolas Brunner $^{3}$, Antonio Acín ${ }^{1,4 \star}$ \\ and Juan P. Torres ${ }^{1,5 \star}$
}

Experimental observations are usually described using theoretical models that make assumptions about the dimensionality of the system under consideration. However, would it be possible to assess the dimension of a completely unknown system only from the results of measurements performed on it, without any extra assumption? The concept of a dimension witness $^{1-6}$ answers this question, as it allows bounding the dimension of an unknown system only from measurement statistics. Here, we report on the experimental demonstration of dimension witnesses in a prepare and measure scenario ${ }^{6}$. We use photon pairs entangled in polarization and orbital angular momentum ${ }^{7-9}$ to generate ensembles of classical and quantum states of dimensions up to 4 . We then use a dimension witness to certify their dimensionality as well as their quantum nature. Our work opens new avenues in quantum information science, where dimension represents a powerful resource $^{10-12}$, especially for device-independent estimation of quantum systems ${ }^{13-16}$ and quantum communications ${ }^{17,18}$.

Dimensionality is one of the most basic and essential concepts in science, inherent to any theory aiming at explaining and predicting experimental observations. In building up a theoretical model, one makes some general and plausible assumptions about the nature and the behaviour of the system under study. The dimension of this system, that is, the number of relevant and independent degrees of freedom needed to describe it, represents one of these initial assumptions. In general, the failure of a theoretical model in predicting experimental data does not necessarily imply that the assumption on the dimensionality is incorrect, because there might exist a different model assuming the same dimension that is able to reproduce the observed data.

A natural question is whether this approach can be reversed and whether the dimension of an unknown system, classical or quantum, can be estimated experimentally. Clearly, the best one can hope for is to provide lower bounds on this unknown dimension. Indeed, every physical system has potentially an infinite number of degrees of freedom, and one can never exclude that they are all necessary to describe the system in more complex experimental arrangements. The goal, then, is to obtain a lower bound on the dimension of the unknown system from the observed measurement data without making any assumption about the detailed functioning of the devices used in the experiment. Besides its fundamental interest, estimating the dimension of an unknown quantum system is also relevant from the perspective of quantum information science, where the Hilbert space dimension is considered as a resource. For instance, using higher-dimensional Hilbert spaces
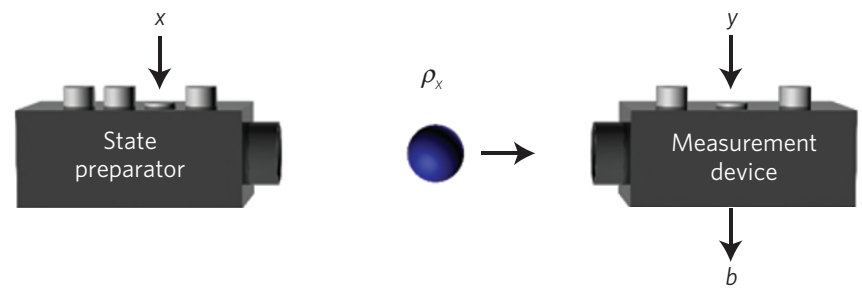

Figure 1 | Sketch of a device-independent test of the dimension of an ensemble of states. Our scenario features two black boxes. First, a state preparator that, on request, prepares the mediating particle in one out of $N$ possible states. Second, a measurement device that performs one out of $M$ possible measurements on the mediating particle.

simplifies quantum logic for quantum computation ${ }^{10}$, enables the optimal realization of information-theoretic protocols ${ }^{11,19,20}$ and allows for lower detection efficiencies in Bell experiments ${ }^{21,22}$. Moreover, the dimension of quantum systems plays a crucial role in security proofs of standard quantum key distribution schemes that become insecure if the dimension is higher than assumed ${ }^{12}$.

The concept of a dimension witness allows one to establish lower bounds on the dimension of an unknown system in a deviceindependent way, that is, only from the collected measurement statistics. It was first introduced for quantum systems in connection with Bell inequalities in ref. 1, and further developed in refs 2, 3,22-26. Other techniques to estimate the dimension have been developed in scenarios involving random access $\operatorname{codes}^{4}$, or the time evolution of a quantum observable ${ }^{5}$.

More recently, a general framework for the study of this question has been proposed in ref. 6 . In this approach, dimension witnesses are defined in a prepare and measure scenario where an unknown system is subject to different preparations and measurements. One of the advantages of this approach is its simplicity from an experimental viewpoint when compared with previous proposals. The considered scenario consists of two devices (Fig. 1), the state preparator and the measurement device. These devices are seen as black boxes, as no assumptions are made on their functioning. The state preparator prepares a state on request. The box features $N$ buttons that label the prepared state; when pressing button $x$, the box emits a state $\rho_{x}$, where $x \in\{1, \ldots, N\}$. The prepared state is then sent to the measurement device. The measurement box performs a measurement $y \in\{1, \ldots, M\}$ on the state, delivering outcome $b \in\{1, \ldots, K\}$. The experiment is thus described by the probability distribution $P(b \mid x, y)$, giving the probability of obtaining outcome

${ }^{1}$ ICFO-Institut de Ciencies Fotoniques, 08860 Castelldefels (Barcelona), Spain, ${ }^{2}$ Department of Optics, Palacký University, 17. listopadu 12, 77146 Olomouc, Czech Republic, ${ }^{3}$ H.H. Wills Physics Laboratory, University of Bristol, Bristol BS8 1TL, UK, ${ }^{4}$ ICREA-Institució Catalana de Recerca i Estudis Avançats, 08010 Barcelona, Spain, ${ }^{5}$ Department of Signal Theory and Communications, Universitat Politècnica de Catalunya, 08034 Barcelona, Spain. *e-mail: antonio.acin@icfo.es; juanp.torres@icfo.es. 
Table 1 | Classical and quantum bounds for the dimension witness $I_{4}$.

\begin{tabular}{llllll} 
& $C_{2}$ (bit) & $Q_{2}$ (qubit) & $C_{3}$ (trit) & $Q_{3}$ (qutrit) & $C_{4}$ (quart) \\
\hline$I_{4}$ & 5 & 6 & 7 & 7.97 & 9
\end{tabular}

The maximal possible value of $I_{4}$ for classical systems of dimension $d$ is denoted $C_{d}$, and the maximum possible value for quantum systems of dimension $d$ is denoted $Q_{d}$. The witness can be used to discriminate ensembles of classical and quantum states of dimension up to $d=4$. Note that for some values of $d$ we have that $C_{d}<Q_{d}$. Thus, if one assumes a bound on the dimension of the system, the witness can be used to certify its quantum nature.

$b$ when measurement $y$ is performed on the prepared state $\rho_{x}$. The goal is to estimate the minimal dimension that the ensemble $\left\{\rho_{x}\right\}$ must have to be able to describe the observed statistics. Moreover, for a fixed dimension, we also aim at distinguishing sets of probabilities $P(b \mid x, y)$ that can be obtained from ensembles of quantum states, but not from ensembles of classical states. This allows one to guarantee the quantum nature of an ensemble of states under the assumption that the dimensionality is bounded. This quantum certification can be exploited for the design of quantum information protocols ${ }^{17,18}$.

Formally, a probability distribution $P(b \mid x, y)$ admits a quantum $d$-dimensional representation if it can be written in the form

$$
P(b \mid x, y)=\operatorname{tr}\left(\rho_{x} O_{b}^{y}\right)
$$

for some state $\rho_{x}$ and measurement operator $O_{b}^{y}$ acting on $\mathbb{C}^{d}$. We then say that $P(b \mid x, y)$ has a classical $d$-dimensional representation if any state of the ensemble $\left\{\rho_{x}\right\}$ is a classical state of dimension $d$, that is, a probability distribution over classical dits (the equivalent in quantum mechanics is that all states in the ensemble act on $\mathbb{C}^{d}$ and commute pairwise).

A dimension witness for systems of dimension $d$ is defined by a linear combination of the observed probabilities $P(b \mid x, y)$, defined by a tensor of real coefficients $D_{b, x, y}$, such that

$$
\sum_{b, x, y} D_{b, x, y} P(b \mid x, y) \leq w_{d}
$$

for all probabilities with a $d$-dimensional representation, whereas the bound $w_{d}$ can be violated by a set of probabilities whose representation has a dimension strictly larger than $d$. Here we shall focus on a dimension witness of the form of equation (2) recently introduced in ref. 6 , for a scenario consisting of $N=4$ possible preparations and $M=3$ measurements with only two possible outcomes, labelled by $b= \pm 1$ :

$$
I_{4} \equiv E_{11}+E_{12}+E_{13}+E_{21}+E_{22}-E_{23}+E_{31}-E_{32}-E_{41}
$$

where $E_{x y}=P(b=+1 \mid x, y)-P(b=-1 \mid x, y)$. The witness $I_{4}$ can distinguish ensembles of classical and quantum states of dimensions up to $d=4$. All of the relevant bounds are summarized in Table 1 .

To test this witness experimentally, we must generate classical and quantum states of dimension 2 (bits and qubits, respectively), classical and quantum states of dimension 3 (trits and qutrits), and classical states of dimension 4 (quarts). To do so we exploit the angular momentum of photons $s^{7,8}$, which contains a spin contribution associated with the polarization, and an orbital contribution associated with the spatial shape of the light intensity and its phase. Within the paraxial regime, both contributions can be measured and manipulated independently. The polarization of photons is conveniently represented by a two-dimensional Hilbert space, spanned by two orthogonal polarization states (for example, horizontal and vertical). The spatial degree of freedom of light lives in an infinite-dimensional Hilbert space ${ }^{27}$, spanned by paraxial
Laguerre-Gaussian modes. Laguerre-Gaussian beams carry a well-defined orbital angular momentum (OAM) of $m \hbar$ ( $m$ is an integer) per photon that is associated with their spiral wavefronts ${ }^{28}$.

In our experiment, we use both the polarization and the OAM $(m= \pm 1)$ of photons to prepare quantum states of dimension up to 4 , spanned by the orthogonal vectors $|H,+1\rangle$, $|H,-1\rangle,|V,+1\rangle$ and $|V,-1\rangle$, where $|H, \pm 1\rangle(|V, \pm 1\rangle)$ denotes a horizontally (vertically) polarized photon with OAM $m= \pm 1$. We first generate, by means of spontaneous parametric downconversion, pairs of photons (signal and idler) entangled in both polarization and OAM (Fig. 2). The entangled state is of the form $\left|\Psi^{-}\right\rangle_{\text {pol }} \otimes\left|\Psi^{-}\right\rangle_{\text {OAM }}$, where $\left|\Psi^{-}\right\rangle_{\text {pol }}=(1 / \sqrt{2})\left(|H\rangle_{s}|V\rangle_{i}-|V\rangle_{s}|H\rangle_{i}\right)$ and $\left|\Psi^{-}\right\rangle_{\mathrm{OAM}}=(1 / \sqrt{2})\left(|m=1\rangle_{s}|m=-1\rangle_{i}+|m=-1\rangle_{s}|m=1\rangle_{i}\right)$. By performing a projective measurement on the idler photon, we prepare the signal photon in a well-defined state of polarization and OAM. In particular, we project the idler photon on states of the form $\left(\cos \theta|H\rangle_{i}+\sin \theta|V\rangle_{i}\right) \otimes|m= \pm 1\rangle_{i}$, which has the effect of preparing the signal photon in the state $\left(\sin \theta|H\rangle_{s}-\right.$ $\left.\cos \theta|V\rangle_{s}\right) \otimes|m=\mp 1\rangle_{s}$. Thus, the combination of the source of entanglement and the measurement of the idler photon represents the state preparator. The prepared state is encoded on the signal photon that is then measured. The signal photon represents the mediating particle between the state preparator and measurement device of Fig. 1.

To implement a continuous transition from quantum to classical states, a polarization-dependent temporal delay $\tau$ between the signal and idler photons is introduced. If the temporal delay between the photons exceeds their correlation time, the coherence is lost; that is, the off-diagonal terms vanish for all states in the ensemble (see Supplementary Information).

For the sake of clarity, we list the assumptions made when processing the observed data: the statistical behaviour of $P(b \mid x y)$, described by equation (1), is the same at every run of the experiment; the detectors used for the preparation and the measurement are uncorrelated; the observer can freely choose the preparation and measurement in each run; the observed statistics provide a fair sample of the total statistics that would have been observed with perfect detectors. All of these assumptions are standard in any estimation scenario. The value of the dimension witnesses is then calculated from the raw data, that is, from all of the observed coincidences between detection at the preparator and at the measuring device, including dark counts. In the experiment we first generate and measure the four qubit states $\left|\phi_{x}\right\rangle$ given in Fig. 2. The first measurement $(y=1)$ assigns dichotomic measurement results of $b=+1$ and $b=-1$ to horizontally and vertically polarized photons, respectively. The second measurement $(y=2)$ assigns $b=+1$ and $b=-1$ to OAM values of $m=+1$ and $m=-1$, respectively. The third measurement $(y=3)$ assigns $b=+1$ and $b=-1$ to photons polarized at $+45^{\circ}$ and $-45^{\circ}$, respectively. The expected value of the dimension witness of equation (3) for this combination of states and measurements is $I_{4}=3+2 \sqrt{2} \sim 5.83$ (see Supplementary Information). From our experimental data we obtain $I_{4}=5.66 \pm 0.15$. This clearly demonstrates the quantum nature of our two-dimensional system, because classical bits always satisfy $I_{4} \leq 5$.

In the above, the delay between signal and idler photons was set to $\tau=0$. Now we gradually increase this delay to convert a qubit into a classical bit. The measured value of the witness $I_{4}$ then drops below 5, as expected (blue triangles in Fig. 3 ).

Next we generate ensembles of qutrits. The prepared states and the measurements are identical to the previous (qubit) experiment, except that the OAM of state $\left|\phi_{3}\right\rangle$ is now flipped. For $\tau=0$, we obtain a measured value of the witness of $I_{4}=7.57 \pm 0.13$, certifying the presence of a quantum system of dimension (at least) 3. This value is in good agreement with the theoretical prediction of $I_{4}=5+2 \sqrt{2} \sim 7.83$ for this set of states and measurements. 

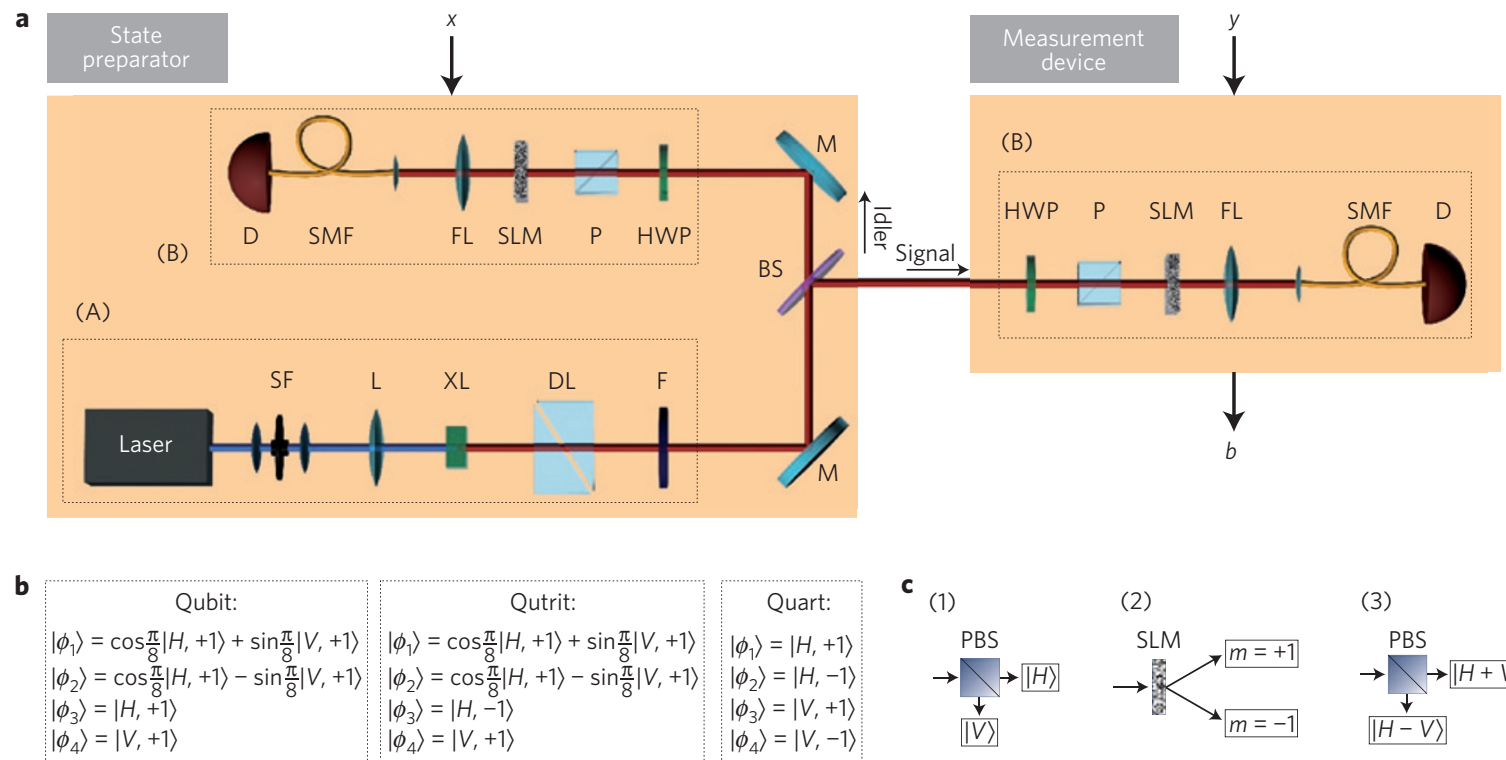

C (1)

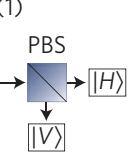

(2)

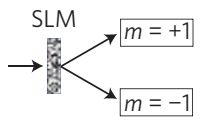

(3)

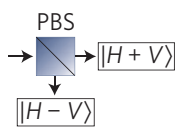

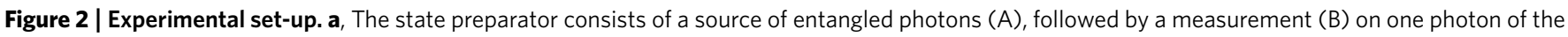

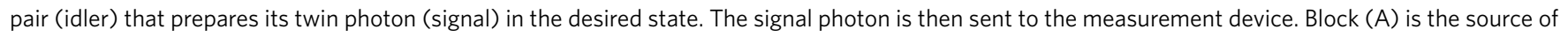
entangled photons. The second harmonic (Inspire Blue, Spectra Physics/Radiantis) at a wavelength of $405 \mathrm{~nm}$ of a Ti:sapphire laser in the picosecond regime (Mira, Coherent) is shaped by a spatial filter (SF) and focused into a 1.5-mm-thick crystal of beta-barium borate, where spontaneous parametric down-conversion takes place. The nonlinear crystal $(\mathrm{XL})$ is cut for collinear type-Il down-conversion so that the generated photons have orthogonal polarizations. Before splitting the signal and idler photon, a polarization-dependent temporal delay $\tau$ is introduced. The delay line (DL) consists of two quartz prisms whose mutual position determines the difference between the propagation times of photons with orthogonal polarizations. Block (B) performs a measurement on the idler photon to prepare the signal photon. It consists of a half-wave plate (HWP), polarizer (P), spatial-light modulator

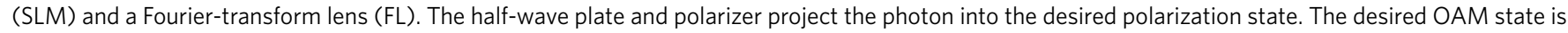
selected by the SLM. SLM encodes computer-generated holograms that transform the $m=+1$ state or $m=-1$ state into the fundamental Laguerre-Gaussian state $\mathrm{LG}_{00}$ (ref. 7) that is coupled into a single-mode fibre (SMF). The measurement device uses an identical block (B) to measure the signal photon. M: mirrors; L: lenses; BS: beam splitter, F: interference filter; D: single-photon counting modules. $\mathbf{b}$, Ensembles of quantum states $\left|\phi_{x}\right\rangle$ $(x=1 \ldots 4)$ prepared in the experiment. c, Measurements performed at the measurement device for qubits and qutrits. In the case of quarts, the three measurements are constructed by combining (1) and (2).

Now, increasing again delay $\tau$ between the photons, the value of the witness drops below 7 . In a certain range of delays, the value of $I_{4}$ remains above the qubit bound of 6 , testifying that at least three dimensions are present (red circles of Fig. 3). In the limit of large delays, the values of the witness are still larger than the bound of $I_{4}=5$ for bits, but below the bound for qubits. This is because the curve was measured with a set a measurements optimized for the qutrit/trit discrimination. This set of measurements is not optimum for the trit/qubit discrimination.

Finally, we prepare classical four-dimensional systems, that is, quarts. Now the first measurement $(y=1)$ assigns the outcome $b=-1$ to vertically polarized photons with OAM $m=-1$, and the outcome $b=+1$ to all of the other orthogonal states. The second measurement $(y=2)$ assigns $b=+1$ and $b=-1$ to horizontally and vertically polarized photons, respectively. The third measurement $(y=3)$ assigns $b=+1$ and $b=-1$ to OAM of $m=+1$ and $m=-1$, respectively. In this case, the expected value of the witness is $I_{4}=9$, which corresponds to the algebraic maximum. Experimentally, we measure $I_{4}=8.57 \pm 0.06$, which violates the qutrit bound of $I_{4}=7.97$ by more than 10 standard deviations. In this case, the values of the witness are independent of the temporal delay $\tau$. This is because the state is here classical (a statistical mixture of orthogonal quantum states) and no superposition is present (green diamonds in Fig. 3).

We have demonstrated how the concept of dimensionality, which is fundamental in science, can be experimentally tested. Using dimension witnesses, we have bounded the dimension of classical and quantum systems only from measurement statistics, without any assumption on the internal working of the devices

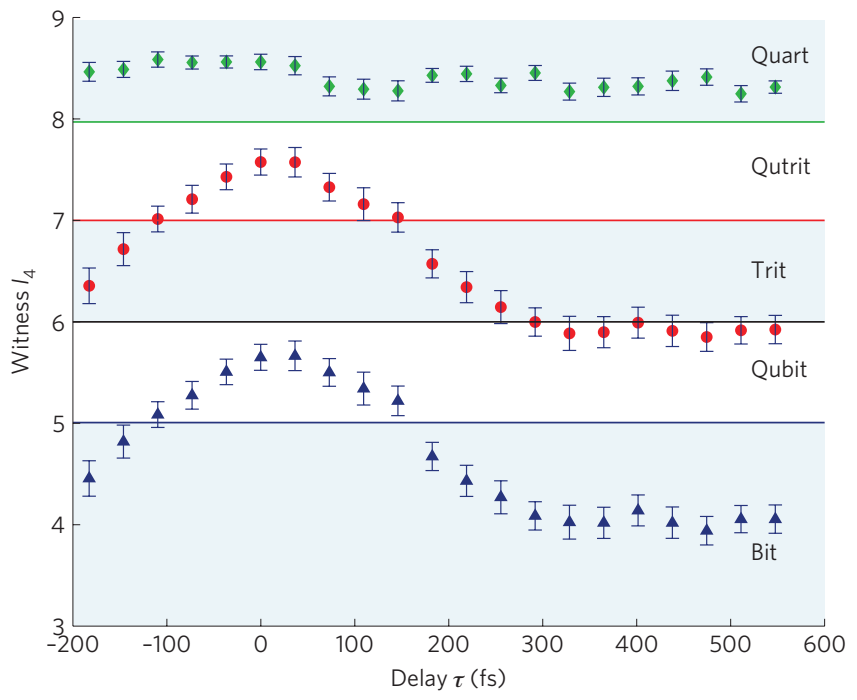

Figure 3 | Dimension witness $I_{4}$ for qubit (blue triangles), qutrit (red circles) and quart (green diamonds) states as a function of temporal delay $\boldsymbol{\tau}$. For delays $\tau>255 \mathrm{fs}$, coherence is lost and quantum superpositions turn into statistical mixtures, that is, classical states. The maximum observed violations for qubit, qutrit and quart states are $5.66 \pm 0.15,7.57 \pm 0.13$ and $8.57 \pm 0.06$, respectively. These values are close to the corresponding theoretical bounds, given in Table 1, which are represented here by the horizontal lines. The error bars plot standard deviations on the value of the witness calculated from the measured data using error propagation rules. 
used in the experiment. Dimension witnesses represent an example of a device-independent estimation technique, in which relevant information about an unknown system is obtained solely from the measurement data. Device-independent techniques ${ }^{13-16}$ provide an alternative approach to existing quantum estimation techniques, such as quantum tomography or entanglement witnesses, which crucially rely on assumptions that may be questionable in complex set-ups, for example, its Hilbert space dimension. Our work demonstrates how the device-independent approach can be employed to experimentally estimate the dimension of an unknown system.

Received 4 March 2012; accepted 3 May 2012; published online 10 June 2012

\section{References}

1. Brunner, N., Pironio, S., Acín, A., Gisin, N., Méthot, A. \& Scarani, V. Testing the dimension of Hilbert spaces. Phys. Rev. Lett. 100, 210503 (2008).

2. Pál, K. \& Vértesi, T. Efficiency of higher-dimensional Hilbert spaces for the violation of Bell inequalities. Phys. Rev. A 77, 042105 (2008).

3. Pérez-García, D., Wolf, M. M., Palazuelos, C., Villanueva, I. \& Junge, M. Unbounded violation of tripartite Bell inequalities. Commun. Math. Phys. 279, 455-486 (2008).

4. Wehner, S., Christandl, M. \& Doherty, A. C. Lower bound on the dimension of a quantum system given measured data. Phys. Rev. A 78, 062112 (2008).

5. Wolf, M. M. \& Pérez-García, D. Assessing quantum dimensionality from observable dynamics. Phys. Rev. Lett. 102, 190504 (2009).

6. Gallego, R., Brunner, N., Hadley, C. \& Acín, A. Device-independent tests of classical and quantum dimensions. Phys. Rev. Lett. 105, 230501 (2010).

7. Molina-Terriza, G., Torres, J. P. \& Torner, L. Twisted photons. Nature Phys. 3, 305-310 (2007).

8. Mair, A., Vaziri, A., Weihs, G. \& Zeilinger, A. Entanglement of the orbital angular momentum states of photons. Nature 412, 313-316 (2001).

9. Dada, A. C., Leach, J., Buller, G. S., Padgett, M. J. \& Andersson, E. Experimental high-dimensional two-photon entanglement and violations of generalized Bell inequalities. Nature Phys. 7, 677-680 (2011).

10. Lanyon, B. et al. Simplifying quantum logic using higher-dimensional Hilbert spaces. Nature Phys. 5, 134-140 (2009).

11. Spekkens, R. W. \& Rudolph, T. Degrees of concealment and bindingness in quantum bit commitment protocols. Phys. Rev. A 65, 012310 (2001).

12. Acín, A., Gisin, N. \& Masanes, Ll. From Bell's theorem to secure quantum key distribution. Phys. Rev. Lett. 97, 120405 (2006).

13. Mayers, D. \& Yao, A. Self testing quantum apparatus. Quant. Inf. Comp. 4, 273-286 (2004).

14. Bardyn, C.-E., Liew, T. C. H., Massar, S., McKague, M. \& Scarani, V. Device-independent state estimation based on Bell's inequalities. Phys. Rev. A 80, 062327 (2009).

15. Bancal, J-D., Gisin, N., Liang, Y-C. \& Pironio, S. Device-independent witnesses of genuine multipartite entanglement. Phys. Rev. Lett. 106, 250404 (2011).
16. Rabelo, R., Ho, M., Cavalcanti, D., Brunner, N. \& Scarani, V. Device-independent certification of entangled measurements. Phys. Rev. Lett. 107, 050502 (2011).

17. Pawlowski, M. \& Brunner, N. Semi-device-independent security of one-way quantum key distribution. Phys. Rev. A 84, 010302 (2011).

18. Li, H-W. et al. Semi-device-independent random-number expansion without entanglement. Phys. Rev. A 84, 034301 (2011).

19. Langford, N. K. et al. Measuring entangled qutrits and their use for quantum bit commitment. Phys. Rev. Lett. 93, 053601 (2004).

20. Molina-Terriza, G., Vaziri, A., Ursin, R. \& Zeilinger, A. Experimental Quantum Coin Tossing. Phys. Rev. Lett. 94, 040501 (2005).

21. Massar, S. Nonlocality, closing the detection loophole, and communication complexity. Phys. Rev. A 65, 032121 (2002).

22. Vértesi, T., Pironio, S. \& Brunner, N. Closing the detection loophole in bell experiments using qudits. Phys. Rev. Lett. 104, 060401 (2010).

23. Vértesi, T. \& Pál, K. Generalized Clauser-Horne-Shimony-Holt inequalities maximally violated by higher-dimensional systems. Phys. Rev. A 77, 042106 (2008).

24. Junge, M., Palazuelos, C., Pérez-García, D., Villanueva, I. \& Wolf, M. M. Operator space theory: A natural framework for bell inequalities. Phys. Rev. Lett. 104, 170405 (2010).

25. Briët, J., Buhrman, H. \& Toner, B. A generalized Grothendieck inequality and nonlocal correlations that require high entanglement. Commun. Math. Phys. 305, 827-843 (2011).

26. Junge, M. \& Palazuelos, C. Large violation of Bell inequalities with low entanglement. Commun. Math. Phys. 306, 695-746 (2011).

27. Molina-Terriza, G., Torres, J. P. \& Torner, L. Management of the orbital angular momentum of light: Preparation of photons in multidimensional vector states of angular momentum. Phys. Rev. Lett. 88, 013601 (2002).

28. Allen, L., Beijersbergen, M. W., Spreeuw, R. J. C. \& Woerdman, J. P. Orbital angular momentum of light and the transformation of Laguerre-Gaussian laser modes. Phys. Rev. A 45, 8185-8189 (1992).

\section{Acknowledgements}

We thank C. Hadley and E. Nagali for contributions in early stages of this work. We acknowledge support from the ERC Starting Grant PERCENT, the EU Projects Q-Essence, QCS and PHORBITECH (FET OPEN grant number 255914), the UK EPSRC, the Project MSM6198959213 of the Czech Ministry of Education, the Spanish projects FIS2010-14830, FIS2010-14831 and Chist-Era DIQIP, an FI Grant of the Generalitat de Catalunya, CatalunyaCaixa, and Fundació Privada Cellex, Barcelona.

\section{Author contributions}

R.G., N.B. and A.A. developed the theory, M.H., R.G., N.B., A.A. and J.P.T. designed the experiment, and M.H., M.M. and J.P.T. performed the experiment. All of the authors contributed to the writing of the manuscript.

\section{Additional information}

The authors declare no competing financial interests. Supplementary information accompanies this paper on www.nature.com/naturephysics. Reprints and permissions information is available online at www.nature.com/reprints. Correspondence and requests for materials should be addressed to A.A. (theory) and J.P.T. (experiment). 